\title{
Hypersurfaces of paraquaternionic space forms ${ }^{1}$
}

\author{
Stere IANUS ${ }^{a}$ and Gabriel Eduard VÎLCU ${ }^{b}$ \\ ${ }^{a}$ Faculty of Mathematics and Computer Science, University of Bucharest, \\ Str. Academiei Nr.14, sector 1, 70109 Bucureşti, Romania \\ E-mail: ianus@gta.math.unibuc.ro \\ ${ }^{b}$ Department of Mathematics and Computer Science, Petroleum-Gas University of Ploieşti, \\ Bulevardul Bucureşti Nr. 39, 100680 Ploieşti, Romania \\ E-mail: gvilcu@mail.upg-ploiesti.ro
}

\begin{abstract}
In this paper we obtain some properties for real lightlike hypersurfaces of paraquaternionic space forms and give an example.
\end{abstract}

2000 MSC: 53C15

\section{Introduction}

It is well-known that the lightlike hypersurfaces are of interest in mathematical physics, owing to their extensive use in general relativity and electromagnetism. The general theory of lightlike submanifolds has been developed by Kupelli [4] and Bejancu-Duggal [1]. In [3], the present authors and R. Mazzocco have begun the study of the real lightlike hypersurfaces of paraquaternionic manifolds. In this paper we obtain new properties for this kind of hypersurfaces and give some obstructions to the existence of lightlike hypersurfaces in paraquaternionic space forms.

\section{$2 \quad$ Lightlike hypersurfaces}

Let $(\bar{M}, \bar{g})$ be a $(m+2)$-dimensional semi-Riemannian manifold with index $q \in\{1,2, \ldots, m+1\}$ and let $(M, g)$ be a hypersurface of $\bar{M}$, with $g=\bar{g}_{\mid M}$. We say [1] that $M$ is a lightlike hypersurface of $\bar{M}$ if $g$ is of constant rank $m$.

If we consider the vector bundle $T M^{\perp}$ whose fibres are:

$$
T_{p} M^{\perp}=\left\{Y_{p} \in T_{p} \bar{M} \mid \bar{g}_{p}\left(X_{p}, Y_{p}\right)=0, \quad \forall X_{p} \in T_{p} M\right\}, \quad \forall p \in M
$$

then we can easily see that $M$ is lightlike if and only if $T M^{\perp}$ is a distribution of rank 1 on $M$.

Let $S(T M)$ be the complementary distribution of $T M^{\perp}$ in $T M$, which is called a screen distribution. This distribution is non-degenerate [1]. Thus we have direct orthogonal sum decomposition $T M=S(T M) \perp T M^{\perp}$. On the other hand, if $S(T M)^{\perp}$ is the orthogonal complementary vector bundle to $S(T M)$ in $T \bar{M}_{\mid M}$, we have direct orthogonal sum $T \bar{M}_{\mid M}=S(T M) \perp S(T M)^{\perp}$.

From [1] we know that, if $(M, g, S(T M))$ is a lightlike hypersurface of $\bar{M}$, then there exists a unique vector bundle $\operatorname{ltr}(T M)$ of rank 1 over $M$ so that for any non-zero section $\xi$ of $T M^{\perp}$ on a coordinate neighborhood $U \subset M$, there exists a unique section $N$ of $l \operatorname{tr}(T M)$ on $U$ satisfying:

$$
\bar{g}(N, \xi)=1, \bar{g}(N, N)=\bar{g}(W, W)=0, \quad \forall W \in \Gamma\left(S(T M)_{\mid U}\right)
$$

\footnotetext{
${ }^{1}$ Presented at the $3^{\text {rd }}$ Baltic-Nordic Workshop "Algebra, Geometry, and Mathematical Physics", Göteborg, Sweden, October 11-13, 2007.
} 
The vector bundle $\operatorname{tr}(T M)$ is called the null transversal vector bundle of $M$ with respect to $S(T M)$. Thus, we have

$$
T \bar{M}_{\mid M}=S(T M) \perp\left(T M^{\perp} \oplus \operatorname{ltr}(T M)\right)=T M \oplus \operatorname{ltr}(T M)
$$

Let $(M, g, S(T M))$ be a lightlike hypersurface of $\bar{M}$. If $\bar{\nabla}$ is the Levi-Civita connection on $\bar{M}$, then we have

$$
\begin{aligned}
& \bar{\nabla}_{X} Y=\nabla_{X} Y+h(X, Y), \quad \forall X, Y \in \Gamma(T M) \\
& \bar{\nabla}_{X} V=-A_{V} X+\nabla_{X}^{\perp} V, \quad \forall X \in \Gamma(T M), \quad \forall V \in \Gamma(\operatorname{ltr}(T M))
\end{aligned}
$$

where $\nabla_{X} Y, A_{V} X \in \Gamma(T M)$ and $h(X, Y), \nabla_{X}^{\perp} V \in \Gamma(l \operatorname{tr}(T M))$.

$\nabla$ is a symmetric linear connection on $M$ called an induced linear connection, $\nabla^{\perp}$ is a linear connection on the null transversal bundle $\operatorname{ltr}(T M), h$ is a $\Gamma(\operatorname{lt}(T M))$-valued symmetric bilinear form and $A_{V}$ is the shape operator of $M$ with respect to $V$. If $\{\xi, N\}$ is a pair of sections on $U \subset M$ as above, then we define a symmetric $F(U)$-bilinear form $B$ and a 1-form $\tau$ on $U$ by

$$
B(X, Y)=\bar{g}(h(X, Y), \xi), \quad \tau(X)=\bar{g}\left(\nabla_{X}^{\perp} N, \xi\right), \quad \forall X, Y \in \Gamma\left(T M_{\mid U}\right)
$$

Thus, from (2.1) and (2.2), locally we have

$$
\begin{aligned}
& \bar{\nabla}_{X} Y=\nabla_{X} Y+B(X, Y) N \\
& \bar{\nabla}_{X} N=-A_{N} X+\tau(X) N
\end{aligned}
$$

If we denote by $P$ the projection of $T M$ on $S(T M)$, we have the decompositions:

$$
\begin{aligned}
\nabla_{X} P Y & =\nabla_{X}^{*} P Y+C(X, P Y) \xi \\
\nabla_{X} \xi & =-A_{\xi}^{*} X+\epsilon(X) \xi
\end{aligned}
$$

where $\nabla_{X}^{*} P Y, A_{\xi}^{*} X \in S(T M)$ and $\epsilon$ is a 1 -form on $U$. Moreover, it is easy to see that $\epsilon=-\tau$.

\section{Real lightlike hypersurfaces of paraquaternionic manifolds}

Let $\bar{M}$ be a smooth manifold. We say that a rank-3 subbundle $\sigma$ of $\operatorname{End}(T \bar{M})$ is an almost paraquaternionic structure on $\bar{M}$ if a local basis $\left\{J_{1}, J_{2}, J_{3}\right\}$ exists on sections of $\sigma$, such that for all $\alpha \in\{1,2,3\}$ we have

$$
J_{\alpha}^{2}=-\epsilon_{\alpha}, \quad J_{\alpha} J_{\alpha+1}=-J_{\alpha+1} J_{\alpha}=\epsilon_{\alpha+2} J_{\alpha+2} \quad(\text { indices mod } 3)
$$

where $\epsilon_{1}=1, \epsilon_{2}=\epsilon_{3}=-1$.

Let $(\bar{M}, \bar{g})$ be a semi-Riemannian manifold and $\sigma$ an almost paraquaternionic structure on $\bar{M}$. The metric $\bar{g}$ is said to be adapted to the paraquaternionic structure $\sigma$ if it satisfies $\bar{g}\left(J_{\alpha} X, J_{\alpha} Y\right)=\epsilon_{\alpha} \bar{g}(X, Y)$ for all $\alpha \in\{1,2,3\}$ and for all vector fields $X, Y$ on $M$ and any local basis $\left\{J_{1}, J_{2}, J_{3}\right\}$ of $\sigma$, where $\epsilon_{1}=1, \epsilon_{2}=\epsilon_{3}=-1$. Moreover $(\bar{M}, \bar{g}, \sigma)$ is said to be an almost hermitian paraquaternionic manifold. Moreover, if the Levi-Civita connection of $\bar{g}$ satisfies the following conditions for all $\alpha \in\{1,2,3\}$ :

$$
\bar{\nabla}_{X} J_{\alpha}=-\epsilon_{\alpha}\left[\omega_{\alpha+2}(X) J_{\alpha+1}-\omega_{\alpha+1}(X) J_{\alpha+2}\right] \quad \text { (indices mod 3) }
$$

for any vector field $X$ on $\bar{M}, \omega_{1}, \omega_{2}, \omega_{3}$ being local 1-forms over the open for which $\left\{J_{1}, J_{2}, J_{3}\right\}$ is a local basis of $\sigma$ and $\epsilon_{1}=1, \epsilon_{2}=\epsilon_{3}=-1$, then $(\bar{M}, \bar{g}, \sigma)$ is said to be a paraquaternionic Kähler manifold (see [2]). 
Let $\left(\bar{M}^{4 m}, \bar{g}, \sigma\right)$ be an almost hermitian paraquaternionic manifold and let $(M, g)$ be a real lightlike hypersurface of $\bar{M}$. We remark that we can choose the screen distribution $S(T M)$ such that it contains $J_{\alpha}(T M)^{\perp}$ as a vector subbundle, because we have: $g\left(J_{\alpha} \xi, \xi\right)=0, \quad \forall \xi \in$ $\Gamma\left(T M_{\mid U}^{\perp}\right), J_{\alpha} \xi$ are tangent to $M$ and thus $J_{\alpha}(T M)^{\perp}$ is a 3 -rank distribution on $M$ such that $J_{\alpha}(T M)^{\perp} \cap T M^{\perp}=\{0\}$.

If $\{\xi, N\}$ is a pair of sections on $U \subset M$ as in above section, then $\bar{g}\left(J_{\alpha} N, \xi\right)=0=\bar{g}\left(J_{\alpha} N, N\right)$, and we deduce that $J_{\alpha} N \in \Gamma(S(T M))$. It is easy to see that $J_{\alpha}\left(T M^{\perp}\right) \oplus J_{\alpha} N$ is a vector subbundle of $S(T M)$ of rank 6 . Then there exists non-degenerate distribution $D_{0}$ on $M$ such that $S(T M)=\left\{D_{1} \oplus D_{2}\right\} \perp D_{0}$, where $D_{1}=J_{1} \xi \perp J_{2} \xi \perp J_{3} \xi$ and $D_{2}=J_{1} N \perp J_{2} N \perp J_{3} N$.

We remark that the distribution $D_{0}$ is invariant with respect to $J_{\alpha}$ for all $\alpha \in\{1,2,3\}$ (see [3]). We consider now the local lightlike vector fields: $V_{\alpha}=-J_{\alpha} N, U_{\alpha}=-J_{\alpha} \xi$ for all $\alpha \in\{1,2,3\}$. Then any local vector field on $M$ is expressed as follows:

$$
X=S X+\sum_{\alpha=1}^{3} \epsilon_{\alpha} f_{\alpha}(X) V_{\alpha}
$$

where $S$ is the projection on the almost paraquaternionic distribution $D=\left\{T M^{\perp} \perp D_{1}\right\} \perp D_{0}$ and $f_{\alpha}$ are 1-forms locally defined on $M$ by: $f_{\alpha}(X)=g\left(X, U_{\alpha}\right)$, for all $\alpha \in\{1,2,3\}$.

By using (3.2) we derive:

$$
J_{\alpha} X=\Phi_{\alpha} X+f_{\alpha}(X) N
$$

where $\Phi_{\alpha} X$ are the tangential components of $J_{\alpha} X$ for all $\alpha \in\{1,2,3\}$.

Theorem 3.1. Let $(\bar{M}, \bar{g}, \sigma)$ be a paraquaternionic Kähler manifold and let $M$ be a lightlike hypersurface of $\bar{M}$. Then $M$ is totally geodesic if and only if: $A_{N} X, A_{\xi}^{*} X, \nabla_{X}^{*} Z_{0} \notin \Gamma\left(D_{2}\right)$, for all $X \in \Gamma(T M), \forall N \in \Gamma(\operatorname{ltr}(T M)), \forall \xi \in \Gamma\left(T M^{\perp}\right), \forall Z_{0} \in \Gamma\left(D_{0}\right)$.

Proof. By using (2.3), (2.4) and (3.1) we obtain for any $X \in \Gamma(T M)$ and $Z_{0} \in \Gamma\left(D_{0}\right)$ :

$$
\begin{aligned}
h\left(X, J_{\alpha} Z_{0}\right)= & -\epsilon_{\alpha}\left[\omega_{\alpha+2}(X) J_{\alpha+1} Z_{0}-\omega_{\alpha+1}(X) J_{\alpha+2} Z_{0}\right] \\
& +J_{\alpha}\left(\nabla_{X}^{*} Z_{0}\right)-C\left(X, Z_{0}\right) U_{\alpha}-B\left(X, Z_{0}\right) V_{\alpha}-\nabla_{X} J_{\alpha} Z_{0}
\end{aligned}
$$

The proof is now complete, since by the definition of a lightlike hypersurface, $M$ is totally geodesic if and only if: $h\left(X, U_{\alpha}\right)=h\left(X, V_{\alpha}\right)=h\left(X, Z_{0}\right)=h(X, \xi)=0, \alpha \in\{1,2,3\}$, for all $Z_{0} \in \Gamma\left(D_{0}\right)$ and $\xi \in \Gamma\left(T M^{\perp}\right)$.

Theorem 3.2. Let $(\bar{M}, \bar{g}, \sigma)$ be a paraquaternionic Kähler manifold and let $M$ be a lightlike hypersurface of $\bar{M}$. Then $D_{0}$ is integrable if and only if: $C(X, Y)=C(Y, X), C\left(X, J_{\alpha} Y\right)=$ $C\left(Y, J_{\alpha} X\right), B\left(X, J_{\alpha} Y\right)=B\left(Y, J_{\alpha} X\right)$ for all $X, Y \in \Gamma\left(D_{0}\right)$ and $\alpha \in\{1,2,3\}$.

Proof. $D_{0}$ is integrable if and only if: $\forall X, Y \in \Gamma\left(D_{0}\right) \Rightarrow[X, Y] \in \Gamma\left(D_{0}\right)$ and from the general decomposition $\left.T M=\left\{\left(D_{1} \oplus D_{2}\right)\right\} \perp D_{0} \perp T M^{\perp}\right\}$ we deduce that $D_{0}$ is integrable if and only if: $\bar{g}([X, Y], N)=\bar{g}\left([X, Y], V_{\alpha}\right)=\bar{g}\left([X, Y], U_{\alpha}\right)=0$ for all $X, Y \in \Gamma\left(D_{0}\right), N \in \Gamma(\operatorname{ltr}(T M))$ and $\alpha \in\{1,2,3\}$. For $X, Y \in \Gamma\left(D_{0}\right)$ and $N \in \Gamma(\operatorname{ltr}(T M))$, by using (2.5) we deduce:

$$
\bar{g}([X, Y], N)=C(X, Y)-C(Y, X)
$$

From (2.5) and (3.1), we obtain:

$$
\bar{g}\left([X, Y], V_{\alpha}\right)=C\left(X, J_{\alpha} Y\right)-C\left(Y, J_{\alpha} X\right), \quad \forall \alpha \in\{1,2,3\}
$$

and similarly we deduce

$$
\bar{g}\left([X, Y], U_{\alpha}\right)=B\left(X, J_{\alpha} Y\right)-B\left(Y, J_{\alpha} X\right), \quad \forall \alpha \in\{1,2,3\}
$$

The proof is now complete from (3.3), (3.4) and (3.5). 
Corollary 3.1. Let $(\bar{M}, \bar{g}, \sigma)$ be a paraquaternionic Kähler manifold and let $M$ be a totally geodesic lightlike hypersurface of $\bar{M}$. Then the distribution $D$ is parallel.

\section{Non-existence of real lightlike hypersurfaces in paraquater- nionic space form}

Let $(\bar{M}, \bar{g}, \sigma)$ be a paraquaternionic Kähler manifold and let $X$ be a non-lightlike vector on $\bar{M}$. Then the 4-plane spanned by $\left\{X, J_{1} X, J_{2} X, J_{3} X\right\}$, denoted by $P Q(X)$, is called a paraquaternionic 4-plane. Any 2-plane in $P Q(X)$ is called a paraquaternionic plane. A paraquaternionic Kähler manifold is said to be a paraquaternionic space form if its paraquaternionic sectional curvatures are equal to a constant.

We recall now the following technical results [3].

Lemma 4.1. Let $(\bar{M}, \bar{g}, \sigma)$ be a paraquaternionic Kähler manifold and let $M$ be a lightlike hypersurface of $\bar{M}$. Then we have for all $\alpha \in\{1,2,3\}$ :

$$
\begin{aligned}
\left(\nabla_{X} \Phi_{\alpha}\right) Y & =-\epsilon_{\alpha}\left[\omega_{\alpha+2}(X) \Phi_{\alpha+1}(Y)-\omega_{\alpha+1}(X) \Phi_{\alpha+2}(Y)\right]+f_{\alpha}(Y) A_{N} X-B(X, Y) V_{\alpha} \\
\left(\nabla_{X} f_{\alpha}\right) Y & =-\epsilon_{\alpha}\left[f_{\alpha+1}(Y) \omega_{\alpha+2}(X)-f_{\alpha+2}(Y) \omega_{\alpha+1}(X)\right]-B\left(X, \Phi_{\alpha} Y\right)-f_{\alpha}(Y) \tau(X)
\end{aligned}
$$

(indices mod 3) for all $X, Y \in \Gamma(T M)$.

Let $(M, g)$ be a lightlike hypersurface of a semi-Riemannian manifold $(\bar{M}, \bar{g})$. The null sectional curvature of $M$ at $p \in M$ with respect to $\xi_{p}$, is the real number $K_{\xi_{p}}(M)$ defined by:

$$
K_{\xi_{p}}(M)=\frac{R\left(X_{p}, \xi_{p}, X_{p}, \xi_{p}\right)}{g\left(X_{p}, X_{p}\right)}
$$

where $X_{p}$ is non-null vector in $T_{p} M$.

Theorem 4.1. There are no real lightlike hypersurfaces with positively or negatively null sectional curvature of paraquaternionic space forms.

Proof. Let $\bar{M}(c)$ be a paraquaternionic space form and let $M$ be a real lightlike hypersurface of $\bar{M}(c)$. By using (4.2) and (4.3) we obtain:

$$
K_{\xi}(M)=\frac{3 c}{4\|X\|^{2}} \sum_{\alpha=1}^{3} \epsilon_{\alpha} f_{\alpha}^{2}(X)
$$

If we take now $X \in \Gamma\left(D_{0}\right)$ we deduce that $K_{\xi}(M)=0$, which proves our assertion.

Theorem 4.2. There are no real lightlike hypersurfaces with positively or negatively Ricci curvature of paraquaternionic space forms.

Proof. Le $M$ be a real lightlike hypersurface of a paraquaternionic space form $\bar{M}(c)$. We have:

$$
\begin{aligned}
\operatorname{Ric}(X, Y)= & \sum_{i=1}^{4 m-8} g\left(Z_{i}, Z_{i}\right) g\left(R\left(X, Z_{i}\right) Y, Z_{i}\right)+g(R(X, \xi) Y, N)+ \\
& +\sum_{\alpha=1}^{3}\left[g\left(R\left(X, U_{\alpha}\right) Y, V_{\alpha}\right)+g\left(R\left(X, V_{\alpha}\right) Y, U_{\alpha}\right)\right]
\end{aligned}
$$

where $\left\{\left\{Z_{i}\right\}_{i=\overline{1,4 m-8}},\left\{U_{\alpha}\right\}_{\alpha=\overline{1,3}},\left\{V_{\alpha}\right\}_{\alpha=\overline{1,3}}\right\}$ is a basis of $S(T M)_{\mid U}$. By straightforward computations using (2.3), (2.4), (2.5), (2.6), (4.1) and (4.2) in (4.4) we obtain $\operatorname{Ric}(X, \xi)=0$. 


\section{Example}

We consider the paraquaternionic manifold $\left(\mathbb{R}_{4}^{8}, \bar{g},\left\{J_{1}, J_{2}, J_{3}\right\}\right)$ where the metric $\bar{g}$ and the structures $J_{1}, J_{2}, J_{3}$ are given by

$$
\begin{aligned}
& \bar{g}\left(\left(x_{i}\right)_{i=\overline{1,8}},\left(y_{i}\right)_{i=\overline{1,8}}\right)=-\sum_{i=1}^{4} x_{i} y_{i}+\sum_{i=5}^{8} x_{i} y_{i} \\
& J_{1}\left(\left(x_{i}\right)_{i=1,8}\right)=\left(-x_{2}, x_{1},-x_{4}, x_{3},-x_{6}, x_{5},-x_{8}, x_{7}\right) \\
& J_{2}\left(\left(x_{i}\right)_{i=\overline{1,8}}\right)=\left(-x_{7}, x_{8},-x_{5}, x_{6},-x_{3}, x_{4},-x_{1}, x_{2}\right) \\
& J_{3}\left(\left(x_{i}\right)_{i=1,8}\right)=\left(x_{8}, x_{7}, x_{6}, x_{5}, x_{4}, x_{3}, x_{2}, x_{1}\right)
\end{aligned}
$$

We define now a hypersurface $M$ of $\left(\mathbb{R}_{4}^{8}, \bar{g},\left\{J_{1}, J_{2}, J_{3}\right\}\right)$ by

$$
f\left(\left(t_{i}\right)_{i=\overline{1,7}}\right)=\left(t_{1}, t_{2}, t_{1}+t_{3}, t_{4}+t_{5}, t_{3}+t_{5}+t_{6}, t_{6}+t_{7}, t_{7}, t_{2}\right)
$$

Thus the tangent space $T M$ is spanned by $\left\{W_{i}\right\}_{i=\overline{1,7}}$, where

$$
\begin{array}{lll}
W_{1}=(1,0,1,0,0,0,0,0), & W_{2}=(0,1,0,0,0,0,0,1), & W_{3}=(0,0,1,0,1,0,0,0) \\
W_{4}=(0,0,0,1,0,0,0,0), & W_{5}=(0,0,0,1,1,0,0,0), & W_{6}=(0,0,0,0,1,1,0,0) \\
W_{7}=(0,0,0,0,0,1,1,0) & &
\end{array}
$$

If $X=\left(x_{1}, x_{2}, x_{3}, x_{4}, x_{5}, x_{6}, x_{7}, x_{8}\right) \in T M^{\perp}$, from conditions $\bar{g}\left(X, W_{i}\right)=0(i \in\{1, \ldots, 7\})$ we obtain

$$
x_{1}=x_{3}=x_{4}=x_{5}=x_{6}=x_{7}=0, \quad x_{2}=x_{8}
$$

Hence,

$$
X=\left(0, x_{2}, 0,0,0,0,0, x_{2}\right)=x_{2} W_{2}
$$

Thus $T M^{\perp}=S p\left(W_{2}\right)$ and $M$ is lightlike hypersurface of $\mathbb{R}_{4}^{8}$.

\section{Acknowledgement}

Research was supported by grant D11-22 CEEX 2006-2008.

\section{References}

[1] A. Bejancu and K. L. Duggal. Lightlike submanifolds of semi-Riemannian manifolds and its applications. Kluwer, Dortrecht, 1996.

[2] E. Garcia-Rio, Y. Matsushita, and R. Vasquez-Lorenzo. Paraquaternionic Kähler manifolds. Rocky Mountain J. Math. 31 (2001), 237-260.

[3] S. Ianuş, R. Mazzocco, and G. E. Vîlcu. Real lightlike hypersurfaces of paraquaternionic Kähler manifolds. Mediterr. J. Math. 3 (2006), 581-592.

[4] N. Kupelli. Singular semi-Riemann geometry. Kluwer, Dortrecht, 1996.

[5] S. Marchiafava. Submanifolds of (para)-quaternionic Kähler manifolds. Note Mat. 2008 (to be published).

[6] B. Şahin and R. Guneş. Lightlike real hypersurfaces of indefinite quaternion Kähler manifolds. J. Geom. 75 (2002), 151-163. 